\section{Ekbom's Syndrome as the First Manifestation of Diabetes Mellitus}

To the Editor: Ekbom's syndrome is a rare and mostly unknown psychiatric disorder that is characterized by the fixed and false conviction of being infested with parasites. ${ }^{1}$ Patients frequently experience tactile, visual, haptic, or auditory hallucinations, giving detailed descriptions of the characteristics and the activities of the offending parasites. Insomnia is a common complaint as the patients are frequently quite disturbed by the parasites. ${ }^{2}$ It can be primary or secondary to several medical and psychiatric disorders or to the use of prescribed or illicit drugs. ${ }^{3}$

\section{Case Report}

A 62-year-old woman with a medical history of congenital idiopathic mental retardation and arterial hypertension presented with tactile, haptic, and visual hallucinations of bugs over the head and members. She reported that they would crawl and bite her arms even at night, preventing her from sleeping. She tried to catch them and show to the physician. She explained these symptoms in a delusional manner, saying that she had been previously bitten by a fly which first inoculated her with the eggs that originated the bugs.

An analytic study showed fasting glucose of $283 \mathrm{mg} / \mathrm{dL}$, with no other abnormality, including blood count, vitamin $\mathrm{B}_{12}$ and folic acid levels, liver, renal, and thyroid functions. A brain MRI was normal. The patient was admitted and treated with pimozide, gliclazide, and metformin. When hospitalized, she brought a napkin that she said was full with bugs and made a drawing of the insects she thought were infesting her. She was discharged 40 days later with discrete improvement of the delusional ideas and fasting glucose levels ranging from 112 to 140 $\mathrm{mg} / \mathrm{dL}$.

\section{Discussion}

Ekbom's syndrome was first methodically described by Karl Axel Ekbom in 1938. ${ }^{4}$ It can occur at any age, though the average is in the fifth decade, particularly among females. ${ }^{3}$ There are several medical disorders which have been found to be related to Ekbom's syndrome, including tuberculosis, syphilis, dermatitis, hypothyroidism, renal and heart failure, hepatic disorders, leukemias, lymphomas, vitamin $\mathrm{B}_{12}$ deficiency, pellagra, diabetes mellitus, dementias, Huntington's disease, cerebral infarction, CNS tumors, multiple sclerosis, and Parkinson's disease. ${ }^{1,2}$

Though there were several cases of Ekbom's syndrome occurring in diabetics, a thorough search on MEDLINE did not retrieve any case of Ekbom's syndrome revealing a previously unknown diabetes mellitus. The contribution of mental retardation to the develop- ment of the syndrome in our patient is unclear, but, because it is more prevalent in such cases, mental retardation might have acted as a predisposing factor.

Our case highlights the need for a thorough laboratory workup in patients with Ekbom's syndrome, as treatable conditions can be found and properly managed.

Joana Mesquita, M.D.

Sónia Simões, M.D.

Psychiatry and Mental Health

Department, Hospital de São

Marcos, Braga, Portugal

Álvaro Machado, M.D.

Neurology Department, Hospital de São Marcos, Braga, Portugal

Luísa Siva, M.D.

Psychiatry and Mental Health

Department, Hospital de São

Marcos, Braga, Portugal

\section{References}

1. Sadock BJ, Sadock VA: Psychocutaneous disorders, in Kaplan and Sadock's Comprehensive Textbook of Psychiatry, 8th ed, vol 2. Philadelphia, Lippincott Williams \& Wilkins, 2005, pp 2169-2170

2. Aw DCW, Thong JY, Chan HL: Delusional parasitosis: case series of 8 patients and review of the literature. Ann Acad Med Singapore 2004; 33:89-94

3. Le L, Gonski PN: Delusional parasitosis mimicking cutaneous infestation in elderly patients. Med J Aust 2003; 179: 209-210

4. Mahler C, MacQueen G, Samaan Z: A postmenopausal woman presenting with Ekbom syndrome associated with recurrent depressive disorder: a case report. Cases J 2008; 1:54 